\title{
Adaptive Output Feedback Stabilization of Nonholonomic Systems with Nonlinear Parameterization
}

\author{
Yanling Shang, ${ }^{1}$ Ye Yuan, ${ }^{2,3}$ and Fushun Yuan ${ }^{2}$ \\ ${ }^{1}$ School of Software, Anyang Normal University, Anyang 455000, China \\ ${ }^{2}$ School of Mathematics and Statistics, Anyang Normal University, Anyang 455000, China \\ ${ }^{3}$ School of Mathematics and Physics, Suzhou University of Science and Technology, Suzhou 215009, China \\ Correspondence should be addressed to Yanling Shang; hnnhsyl@126.com
}

Received 18 April 2014; Accepted 24 July 2014; Published 12 August 2014

Academic Editor: Janusz Brzdek

Copyright (C) 2014 Yanling Shang et al. This is an open access article distributed under the Creative Commons Attribution License, which permits unrestricted use, distribution, and reproduction in any medium, provided the original work is properly cited.

\begin{abstract}
This paper investigates the problem of adaptive output feedback stabilization for a class of nonholonomic systems with nonlinear parameterization and strong nonlinear drifts. A parameter separation technique is introduced to transform nonlinearly parameterized system into a linear-like parameterized system. Then, by using the integrator backstepping approach based on observer and parameter estimator, a constructive design procedure for output feedback adaptive control is given. And a switching strategy is developed to eliminate the phenomenon of uncontrollability. It is shown that, under some conditions, the proposed controller can guarantee that all the system states globally converge to the origin, while other signals remain bounded. An illustrative example is also provided to demonstrate the effectiveness of the proposed scheme.
\end{abstract}

\section{Introduction}

Control of nonholonomic systems has received a great deal of attention over the last few years. It has been shown in [1] that mechanical systems with nonholonomic constraints such as mobile robots and wheeled vehicle can be either locally or globally converted to the so-called chained form under a coordinate transformation and a control mapping. As a result, the chained form has been used as a canonical form in analysis and control design for nonholonomic systems. Due to the nonsatisfaction of Brockett's necessary condition [2], nonholonomic systems cannot be asymptotically stabilized by stationary continuous state-feedback, although it is controllable. To overcome this difficulty, a number of approaches have been proposed; see the review paper [3] and references therein. The proposed solutions for stabilization nonholonomic systems include discontinuous feedback, time-varying feedback, and hybrid control laws. Using there valid approaches, the robustness issue for the asymptotic and exponential stability properties has been extensively studied [4-20].
However, it should be noticed that most of these papers were concerned with the systems with linear parameterization. Since nonlinear parameterization is exceptionally difficult to estimate, there are very few reports in literature for adaptive control of nonlinearly parameterized nonholonomic systems. Exceptions include [21-23] (and some references therein), where the authors consider that the whole state vector is measurable and adaptive control for chained form systems with nonlinear parameterization using state feedback.

In this paper, the case of only partial state vector being measurable and adaptive control for chained form systems with nonlinear parameterization by output feedback is considered. The contributions of this paper are listed as follows: (i) a new observer design method is proposed, and, based on the observer, the unmeasurable states of the system involved are reconstructed; (ii) using parameter separation technique [24], the parameters nonlinearities are solved and the resulting adaptive regulator is of minimum dimension (1D) independent of the parameter dimension; (iii) output feedback adaptive control based switching strategy is adopted 
to handle the technical problem of uncontrollability at $x_{0}\left(t_{0}\right)=0$, which prevents the finite escape of systems and guarantees that all the states converge to the origin and other signals are bounded.

The rest of this paper is organized as follows. In Section 2 preliminary knowledge and the problem formulation are given. Section 3 presents the state-scaling technique and the backstepping design procedure, while Section 4 provides the switching control strategy and the main result. Section 5 gives a simulation example to illustrate the theoretical finding of this paper. Finally, concluding remarks are proposed in Section 6.

\section{Problem Formulation}

In this paper, we present an output feedback adaptive control design procedure for a class of uncertain chained form systems with nonlinear parameterization:

$$
\begin{gathered}
\dot{x}_{0}=u_{0}+f_{1}\left(x_{0}, \theta\right) \\
\dot{x}_{i}=u_{0} x_{i+1}+f_{i}\left(x_{0}, x, \theta\right) \quad 1 \leq i \leq n-1 \\
\dot{x}_{n}=u_{1}+f_{n}\left(x_{0}, x, \theta\right) \\
y=\left(x_{0}, x_{1}\right)^{T},
\end{gathered}
$$

where $\left(x_{0}, x\right)^{T}=\left(x_{0}, x_{1}, \ldots, x_{n}\right)^{T} \in R^{n+1}, u=\left(u_{0}, u_{1}\right)^{T} \in R^{2}$, and $y \in R^{2}$ are the system state, control input, and system measurable output, $f_{i}, i=0,1, \ldots, n$ are continuous functions of their arguments, and $\theta \in R^{p}$ is an unknown constant vector. The function $f_{i}$ is called the nonlinear drifts of the system (1).

The objective of this paper is to design an output feedback adaptive stabilization control in the form

$$
\begin{array}{ll}
\dot{\hat{x}}=\vartheta(\hat{x}, y), & \dot{\mu}=\nu\left(x_{0}, x, \mu\right) \\
u_{0}=u_{0}(y, \mu), & u_{1}=u_{1}(y, \mu)
\end{array}
$$

such that all signals of the closed loop system are bounded. Furthermore, the asymptotic regulation of the states is achieved; that is, $\lim _{t \rightarrow \infty}\left(\left|x_{0}(t)\right|+|x(t)|\right)=0$.

A full characterization of the class of system (1) is given by the following assumption, which will be the base of the coming control design and performance analysis.

Assumption 1. For $f_{0}$, there exists a smooth nonnegative function $\varphi_{0}$ such that

$$
\left|f_{0}\left(x_{0}, \theta\right)\right| \leq\left|x_{0}\right| \varphi_{0}\left(x_{0}, \theta\right) .
$$

For $1 \leq i \leq n$, there are smooth nonnegative function $\varphi_{i}$ such that

$$
\left|f_{i}\left(x_{0}, x, \theta\right)\right| \leq\left|x_{1}\right| \varphi_{i}(y, \theta)
$$

Remark 2. Assumptions 1 implies that $f_{0}(0, \theta) \equiv 0$ and $f_{i}(0,0, \theta) \equiv 0$, that is, the origin is the equilibrium point of system (1).
Lemma 3 (see [24]). For any real-valued continuous function $f(x, y)$, where $x \in R^{m}, y \in R^{n}$, there are smooth scalar functions $a(x) \geq 0, b(y) \geq 0, c(x) \geq 1$ and $d(y) \geq 1$, such that

$$
\begin{gathered}
|f(x, y)| \leq a(x)+b(y) \\
|f(x, y)| \leq c(x) d(y) .
\end{gathered}
$$

By Assumption 1 and Lemma 3, we easily obtain the following lemma.

Lemma 4. For $1 \leq i \leq n$, there are smooth nonnegative function $\bar{\varphi}_{i}$ and unknown constant $\Theta_{i} \geq 1$ such that

$$
\begin{gathered}
\left|f_{0}\left(x_{0}, \theta\right)\right| \leq\left|x_{0}\right| \bar{\varphi}_{0}\left(x_{0}\right) \Theta_{0} \\
\left|f_{i}\left(x_{0}, x, \theta\right)\right| \leq\left|x_{1}\right| \bar{\varphi}_{i}(y) \Theta_{i} .
\end{gathered}
$$

\section{Adaptive Output Feedback Control Design}

In this section, we focus on designing the control input $u_{1}$ via output feedback provided that $x_{0}\left(t_{0}\right) \neq 0$. The case where the initial $x_{0}\left(t_{0}\right)=0$ will be treated in Section 4 . The special structure of the system (1) suggests that we should design the control inputs $u_{0}$ and $u_{1}$ in two separate stages.

Let $\widetilde{\Theta}_{0}=\Theta_{0}-\widehat{\Theta}_{0}$, where $\widehat{\Theta}_{0}$ is an estimate of $\Theta_{0}$. Assumption 1 leads us to choose the control law $u_{0}$ as

$$
\begin{gathered}
u_{0}\left(x_{0}, \widehat{\Theta}_{0}\right)=x_{0} g_{0}\left(x_{0}, \widehat{\Theta}_{0}\right) \\
g_{0}\left(x_{0}, \widehat{\Theta}_{0}\right)=-\left[\bar{\varphi}_{0} \widehat{\Theta}_{0}+\sqrt{2 k_{0}^{2}+2\left(m_{0} \bar{\varphi}_{0}+\bar{\varphi}_{0} \widehat{\Theta}_{0}\right)^{2}}\right],
\end{gathered}
$$

where $k_{0}$ and $m_{0}$ are positive design parameters to be specified later.

Remark 5. Note the control $u_{0}$ is an uncertain version of Sontag formula [25]. It is used to stabilize $x_{0}$ with uncertainties. Because the particular choice of $(8), g_{0}\left(x_{0}, \widehat{\Theta}_{0}\right) \neq 0$ is guaranteed irregardless of the values of $x_{0}$. Hence, $1 / g_{0}$ is well defined.

Consider the Lyapunov function candidate

$$
V_{0}=\frac{1}{2} x_{0}^{2}+\frac{1}{2} \widetilde{\Theta}_{0}^{2} .
$$

From (7) and (8) and Assumption 1, we have

$$
\begin{aligned}
\dot{V}_{0} & =x_{0}\left[u_{0}+f_{0}\left(x_{0}, \theta\right)\right]-\widetilde{\Theta}_{0} \dot{\widehat{\Theta}}_{0} \\
& \leq g_{0} x_{0}^{2}+x_{0}^{2} \bar{\varphi}_{0} \Theta-\widetilde{\Theta}_{0} \dot{\widehat{\Theta}}_{0} .
\end{aligned}
$$

Choose the adaptation law $\widehat{\Theta}_{0}$ as

$$
\dot{\widehat{\Theta}}_{0}=x_{0}^{2} \bar{\varphi}_{0}\left(x_{0}\right)
$$

renders

$$
\dot{V}_{0} \leq-k_{0} x_{0}^{2}
$$


Therefore, $x_{0}$ and $\widetilde{\Theta}_{0}$ (or, equivalently, $\widehat{\Theta}_{0}$ ) are bounded; without loss of generality, we assume $\left|\widetilde{\Theta}_{0}\right| \leq M$, so we can choose design parameter $m_{0}$ in (8) as $m_{0}>M$. Furthermore, we can conclude that $x_{0} \rightarrow 0$ as $t \rightarrow \infty$ by using LaSalle's Invariant Theorem [26].

Under the control law (7), the solution of $x_{0}$-subsystem can be expressed as

$$
x_{0}(t)=x_{0}\left(t_{0}\right) \exp \left(\int_{t_{0}}^{t} \frac{u_{0}+f_{0}}{x_{0}} d s\right)
$$

which implies

$$
\begin{aligned}
\left|x_{0}(t)\right| & \geq\left|x_{0}\left(t_{0}\right)\right| \exp \left(-\int_{t_{0}}^{t}\left|\frac{u_{0}+f_{0}}{x_{0}}\right| d s\right) \\
& \geq\left|x_{0}\left(t_{0}\right)\right| \exp \left(-\int_{t_{0}}^{t} \lambda(s) d s\right),
\end{aligned}
$$

where $\lambda(s)=2 \bar{\varphi}_{0} \widehat{\Theta}_{0}+\sqrt{2 k_{0}+2\left(m_{0} \bar{\varphi}_{0}+\bar{\varphi}_{0} \widehat{\Theta}_{0}\right)^{2}}+k_{0} \bar{\varphi}_{0}$.

Consequently, $x_{0}$ does not cross zero for all $t \in\left(t_{0}, \infty\right)$ provided that $x_{0}\left(t_{0}\right) \neq 0$.

From the above analysis, we can see the $x_{0}$-state in (1) can be globally regulated to zero via $u_{0}$ in (7) as $t \rightarrow \infty$. It is troublesome in controlling the $x$-subsystem via the control input $u_{1}$ in the limit (i.e., $u_{0}=0$ ), the $x$-subsystem is uncontrollable. This problem can be avoided by utilizing the following discontinuous state scaling transformation [4]:

$$
z_{i}=\frac{x_{i}}{x_{0}^{n-i}} \quad 1 \leq i \leq n
$$

Under the new $z$-coordinates, with choice of $u_{0}$ as in (7), the $x$-system is transformed into

$$
\begin{gathered}
\dot{z}_{1}=g_{0} z_{2}-(n-1) g_{0} z_{1}+\frac{f_{1}}{x_{0}^{n-1}} \\
\dot{z}_{i}=g_{0} z_{i+1}-(n-i) g_{0} z_{1}+\frac{f_{i}}{x_{0}^{n-2}} \\
\dot{z}_{n}=u_{1}+f_{n} .
\end{gathered}
$$

It should be noted that the measurement of state $z_{1}$ can be obtained if the to-be-designed control $u_{0}$ is only dependent on output $y$. If $x_{0}(t) \neq 0$ for every $t \geq 0$, the discontinuous state transformation (15) is applicable.

We design the following observer for the system (16):

$$
\begin{gathered}
\dot{\bar{z}}_{1}=g_{0} \widehat{z}_{2}-(n-1) g_{0} \widehat{z}_{1}+\frac{k_{1} u_{0}}{x_{0}}\left(z_{1}-\widehat{z}_{1}\right) \\
\dot{\vec{z}}_{i}=g_{0} \widehat{z}_{i+1}-g_{0} \widehat{z}_{i}+\frac{k_{i} u_{0}}{x_{0}}\left(z_{1}-\widehat{z}_{1}\right) \\
\dot{\vec{z}}_{n}=u_{1}+\frac{k_{n} u_{0}}{x_{0}}\left(z_{1}-\widehat{z}_{1}\right),
\end{gathered}
$$

where $k_{1}, \ldots, k_{n}$ are design parameters to be determined later.
The estimation error $\widetilde{z}=z-\widehat{z}$ satisfies the dynamical equations:

$$
\begin{gathered}
\dot{\tilde{z}}_{1}=g_{0} \widetilde{z}_{2}-(n-1) g_{0} \widetilde{z}_{1}-k_{1} g_{0} \widetilde{z}_{1}+\frac{f_{1}}{x_{0}^{n-1}} \\
\dot{\tilde{z}}_{i}=g_{0} \widetilde{z}_{i+1}-(n-i) g_{0} \widetilde{z}_{i}-k_{i} g_{0} \widetilde{z}_{1}+\frac{f_{i}}{x_{0}^{n-i}} \\
\dot{\tilde{z}}_{n}=-k_{n} g_{0} \widetilde{z}_{1}+f_{n}+\phi_{n}^{d} .
\end{gathered}
$$

The differential equations (18) can be rewritten into the compact form

$$
\dot{\tilde{z}}=g_{0} A \widetilde{z}+\Phi,
$$

where

$$
\begin{gathered}
A=\left(\begin{array}{cccccc}
-k_{1}-n+1 & 1 & 0 & 0 & \cdots & 0 \\
-k_{2} & -n+2 & 1 & 0 & \cdots & 0 \\
\vdots & 0 & -n+3 & 1 & \ddots & 0 \\
\vdots & \vdots & \ddots & \ddots & \ddots & 0 \\
-k_{n-1} & \vdots & \vdots & \ddots & -1 & 1 \\
-k_{n} & 0 & \cdots & \cdots & 0 & 0
\end{array}\right) \\
\Phi=\left(\begin{array}{c}
\frac{f_{1}}{x_{0}^{n-1}} \\
\frac{f_{2}}{x_{0}^{n-2}} \\
\vdots \\
\frac{f_{n-1}}{x_{0}} \\
f_{n}
\end{array}\right) .
\end{gathered}
$$

About matrix $A$ defined by (20), there exists the following lemma.

Lemma 6. The eigenvalues of the matrix $A$ defined by (20) can be arbitrarily assigned by a proper selection of the design parameters $k_{1}, \ldots, k_{n}$.

Proof. The proof can be found in [16] and thus omitted here.

Lemma 7. By Assumption 1 and Lemma 4, we easily obtain that, for every $1 \leq i \leq n$, there exists smooth nonnegative function $\gamma_{i}$ such that

$$
\left|\frac{f_{i}\left(x_{0}, x, \theta\right)}{x_{0}^{n-i}}\right| \leq\left|z_{1}\right| \gamma_{i}\left(x_{0}, z_{1}, \widehat{\Theta}_{0}\right) \Theta_{i}
$$


In view of (18), (19), and (16), the overall system to be controlled can be expressed as

$$
\begin{gathered}
\dot{\vec{z}}=g_{0} A \widetilde{z}+\Phi \\
\dot{z}_{1}=g_{0} \widehat{z}_{2}+g_{0} \widetilde{z}_{2}-(n-1) g_{0} z_{1}+\frac{f_{1}}{x_{0}^{n-1}} \\
\dot{\vec{z}}_{i}=g_{0} \widehat{z}_{i+1}-(n-i) g_{0} \widehat{z}_{i}+k_{i} g_{0} \widetilde{z}_{1} \\
\dot{\vec{z}}_{n}=u_{1}+k_{n} g_{0} \widetilde{z}_{1} .
\end{gathered}
$$

We now turn to the constructive design procedure of the control.

Step 1. This step can be regarded as the initial assignation of the entire design procedure. At this step, we introduce a Lyapunov function for the estimation error $\widetilde{z}$. Define $\Theta=$ $\max \left\{\Theta_{i}, \Theta_{i}^{2}\right\}$ and $\widetilde{\Theta}=\Theta-\widehat{\Theta}$, where $\widehat{\Theta}$ is the estimate of $\Theta$. Consider the Lyapunov function

$$
U_{0}=\widetilde{z}^{T} P \widetilde{z}+\frac{\widetilde{\Theta}^{2}}{2},
$$

where $P$ is the positive definite solution of the Riccati equation

$$
P A+A^{T} P=I_{n} .
$$

Then, taking time-derivation of $U_{0}$ along the solution of (19), we have

$$
\dot{U}_{0}=g_{0}\|\widetilde{z}\|^{2}+2 \widetilde{z}^{T} P \Phi-\widetilde{\Theta} \dot{\widehat{\Theta}}
$$

By Lemma 7 and the expression of $\Phi$ in (20), we have

$$
\|\Phi\|^{2} \leq z_{1}^{2} \bar{\Phi}\left(x_{0}, z_{1}, \widehat{\Theta}_{0}\right) \Theta
$$

where $\bar{\Phi}=\sum_{i=1}^{n} \gamma_{i}^{2}\left(x_{0}, z_{1}, \widehat{\Theta}_{0}\right)$ is a nonnegative smooth function. Then, for the terms on the right-hand side of (25), by completing the square, we have

$$
\begin{aligned}
2 \widetilde{z}^{T} P \Phi & =\varepsilon_{0}^{2}\|\widetilde{z}\|^{2}+\varepsilon_{0}^{-2} \Phi^{T} P P \Phi-\varepsilon_{0}^{2}\left\|\widetilde{z}-\varepsilon_{0}^{-2} P \Phi\right\|^{2} \\
& \leq \varepsilon_{0}^{2}\|\tilde{z}\|^{2}+\varepsilon_{0}^{-2}\|P\|^{2} z_{1}^{2} \bar{\Phi}^{2} \Theta
\end{aligned}
$$

where $\varepsilon_{0}$ is a positive design parameter to be specified later.

Substituting (27) into (25) leads to

$$
\dot{U}_{0}=\left(g_{0}+\varepsilon_{0}^{2}\right)\|\widetilde{z}\|^{2}+\Psi_{0}(\widehat{\Theta}+\widetilde{\Theta})-\dot{\widehat{\Theta}} \widetilde{\Theta}
$$

where $\Psi_{0}=\varepsilon_{0}^{-2}\|P\|^{2} z_{1}^{2} \bar{\Phi}^{2}$.

Step 2. Take $V_{1}=U_{0}+z_{1}^{2} / 2$ as the Lyapunov function of this step. Then, by (28) we have

$$
\begin{aligned}
\dot{V}_{1} \leq & \left(g_{0}+\varepsilon_{0}^{2}\right)\|\widetilde{z}\|^{2}+\Psi_{0}(\widehat{\Theta}+\widetilde{\Theta})-\dot{\widehat{\Theta}} \widetilde{\Theta} \\
& +g_{0} z_{1} \widehat{z}_{2}+g_{0} z_{1} \widetilde{z}_{2}+z_{1}^{2} \gamma_{1}(\widetilde{\Theta}+\widehat{\Theta})
\end{aligned}
$$

with the choice of the virtual controller

$$
\begin{aligned}
\widehat{z}_{2}^{*} & =-\frac{1}{g_{0}} z_{1}\left[n+g_{0}^{2} \varepsilon_{1}^{-2}+\left(\varepsilon_{0}^{-2}\|P\|^{2} \bar{\Phi}^{2}+\gamma_{1}\right) \sqrt{\widehat{\Theta}^{2}+1}\right] \\
& =z_{1} \beta_{1}\left(x_{0}, z_{1}, \widehat{\Theta}\right)
\end{aligned}
$$

we have

$$
\begin{aligned}
\dot{V}_{1} \leq & \left(g_{0}+\varepsilon_{0}^{2}+\varepsilon_{1}^{2}\right)\|\widetilde{z}\|^{2}-n z_{1}^{2}+g_{0} z_{1}\left(\widehat{z}_{2}-\widehat{z}_{2}^{*}\right) \\
& +\left(\Psi_{1}-\dot{\widehat{\Theta}}\right)\left(\widetilde{\Theta}+\eta_{1}\right),
\end{aligned}
$$

where $\Psi_{1}=z_{1}^{2}\left(\varepsilon_{0}^{-2}\|P\|^{2} \bar{\Phi}^{2}+\gamma_{1}\right) \geq 0$ and $\eta_{1}=0$.

Step $i(2 \leq I \leq n)$. Suppose at step $i-1$ we have designed a set of smooth virtual controllers $\widehat{z}_{1}^{*}, \ldots, \widehat{z}_{i}^{*}$ defined by

$$
\begin{aligned}
z_{1}^{*} & =0 \\
\widehat{z}_{2}^{*} & =\xi_{1} \beta_{1}\left(x_{0}, \xi_{1}, \widehat{\Theta}_{0}, \widehat{\Theta}\right) \\
& \vdots \\
\widehat{z}_{i}^{*} & =\xi_{i-1} \beta_{i-1}\left(x_{0}, \xi_{1}, \ldots, \xi_{i-1}, \widehat{\Theta}_{0}, \widehat{\Theta}\right) \\
\xi_{1} & =z_{1}-z_{1}^{*} \\
\xi_{2} & =\widehat{z}_{2}-\widehat{z}_{2}^{*} \\
& \vdots \\
\xi_{i} & =\widehat{z}_{i}-\widehat{z}_{i}^{*}
\end{aligned}
$$

with $\beta_{1}\left(x_{0}, \xi_{1}, \widehat{\Theta}\right)>0, \ldots, \beta_{i-1}\left(x_{0}, \xi_{1}, \ldots, \xi_{i-1}, \widehat{\Theta}\right)>0$ being smooth, such that

$$
\begin{aligned}
\dot{V}_{i-1}= & \left(g_{0}+\sum_{k=0}^{i-1} \varepsilon_{k}^{2}\right)\|\tilde{z}\|^{2}-(n-i+2)\left(\xi_{1}^{2}+\cdots+\xi_{i-1}^{2}\right) \\
& +g_{0} \xi_{i-1}\left(\widehat{z}_{i}-\widehat{z}_{i}^{*}\right)+\left(\Psi_{i-1}-\dot{\widehat{\Theta}}\right)\left(\widetilde{\Theta}+\eta_{i-1}\right)
\end{aligned}
$$

where

$$
\begin{aligned}
0 & \leq \Psi_{i-1}\left(x_{0}, \xi_{1}, \ldots, \xi_{i-1}, \widehat{\Theta}_{0}, \widehat{\Theta}\right) \\
& \leq\left(\xi_{1}^{2}+\cdots+\xi_{i-1}^{2}\right) \alpha_{i-1}\left(x_{0}, \xi_{1}, \ldots, \xi_{i-1}, \widehat{\Theta}_{0}, \widehat{\Theta}\right)
\end{aligned}
$$

for $C^{\infty} \alpha_{i-1} \geq 0$

We intend to establish a similar property for $(\widetilde{z}$, $z_{1}, \ldots, \widehat{z}_{i}$ )-subsystem. Consider the Lyapunov function

$V_{i}\left(x_{0}, \xi_{1}, \ldots, \xi_{i}, \widehat{\Theta}_{0}, \widehat{\Theta}\right)=V_{i-1}\left(x_{0}, \xi_{1}, \ldots, \xi_{i-1}, \widehat{\Theta}_{0}, \widehat{\Theta}\right)+\frac{1}{2} \xi_{i}^{2}$ 
Clearly

$$
\begin{aligned}
& \dot{V}_{i}=\left(g_{0}+\sum_{k=0}^{i-1} \varepsilon_{k}^{2}\right)\|\tilde{z}\|^{2}-(n-i+2)\left(\xi_{1}^{2}+\cdots+\xi_{i-1}^{2}\right) \\
& +g_{0} \xi_{i-1}\left(\widehat{z}_{i}-\widehat{z}_{i}^{*}\right)+\left(\Psi_{i}-\dot{\widehat{\Theta}}\right)\left(\widetilde{\Theta}+\eta_{i}\right) \\
& +\xi_{i}\left\{g_{0} \widehat{z}_{i+1}-(n-i) g_{0} \widehat{z}_{i}+k_{i} g_{0} \widetilde{z}_{1}-\frac{\partial z_{i}^{*}}{\partial \widehat{\Theta}_{0}} x_{0}^{2} \bar{\varphi}_{0}\right. \\
& -\frac{\partial \widehat{z}_{i}^{*}}{\partial \widehat{\Theta}} \dot{\widehat{\Theta}}-\frac{\partial z_{i}^{*}}{\partial x_{0}}\left(u_{0}+f_{0}\right) \\
& -\frac{\partial \widehat{z}_{i}^{*}}{\partial z_{1}}\left[g_{0} z_{2}-(n-1) g_{0} z_{1}+\frac{f_{1}}{x_{0}^{n-1}}\right] \\
& \left.-\sum_{j=2}^{i-1} \frac{\partial \widehat{z}_{i}^{*}}{\partial \widehat{z}_{j}}\left[g_{0} \widehat{z}_{j+1}-(n-j) g_{0} \widehat{z}_{j}+k_{j} g_{0} \widetilde{z}_{1}\right]\right\} \text {. }
\end{aligned}
$$

Since $\widehat{z}_{i}^{*}$ is a smooth function and satisfies

$$
\widehat{z}_{i}^{*}\left(x_{0}, 0, \ldots, 0, \widehat{\Theta}_{0}, \widehat{\Theta}\right)=0
$$

therefore there exist some continuous functions $\psi_{i}, 1 \leq i \leq$ $n-1$ such that

$$
\widehat{z}_{i}^{*}\left(x_{0}, \xi_{1}, \ldots, \xi_{i-1}, \widehat{\Theta}_{0}, \widehat{\Theta}\right)=\sum_{j=1}^{i-1} \xi_{k} \psi_{j}\left(x_{0}, \xi_{1}, \ldots, \xi_{i-1}, \widehat{\Theta}_{0}, \widehat{\Theta}\right) \text {. }
$$

After lengthy but simple calculations based on the completion of squares, there is a smooth nonnegative function $\sigma_{i}$ such that

$$
\begin{aligned}
\left|\xi_{i}\right| \mid & (n-i) g_{0} \widehat{z}_{i}-k_{i} g_{0} \widetilde{z}_{1}+\frac{\partial z_{i}^{*}}{\partial \widehat{\Theta}_{0}} x_{0}^{2} \bar{\varphi}_{0} \\
& +\frac{\partial \widehat{z}_{i}^{*}}{\partial \widehat{\Theta}} \dot{\widehat{\Theta}}+\frac{\partial z_{i}^{*}}{\partial x_{0}} u_{0}+\frac{\partial \widehat{z}_{i}^{*}}{\partial z_{1}}\left[g_{0} z_{2}-(n-1) g_{0} z_{1}\right] \\
& +\sum_{j=2}^{i-1} \frac{\partial \widehat{z}_{i}^{*}}{\partial \widehat{z}_{j}}\left[g_{0} \widehat{z}_{j+1}-(n-j) g_{0} \widehat{z}_{j}+k_{j} g_{0} \widetilde{z}_{1}\right] \mid \\
\leq & \varepsilon_{i}^{2}\|\tilde{z}\|^{2}+\frac{1}{4} \sum_{j=1}^{i-1} \xi_{j}^{2}+\xi_{i}^{2} \sigma_{i}\left(x_{0}, \xi_{1}, \ldots, \xi_{i}, \widehat{\Theta}_{0}, \widehat{\Theta}\right),
\end{aligned}
$$

where $\varepsilon_{i}$ is a positive design parameter to be specified later.

Using (38) and Lemma 4, we have

$$
\left|\frac{\partial z_{i}^{*}}{\partial x_{0}} f_{0}\right| \leq \sum_{j=1}^{i}\left|\xi_{j}\right| \rho_{i}\left(x_{0}, \xi_{1}, \ldots, \xi_{i}, \widehat{\Theta}_{0}, \widehat{\Theta}\right) \Theta_{0}
$$

where $\rho_{i} \geq 0$ is a smooth function.
By Lemma 7, there is a smooth function $\omega_{i} \geq 0$ such that

$$
\left|\frac{\partial \widehat{z}_{i}^{*}}{\partial z_{1}}\right|\left|\frac{f_{1}}{x_{0}^{n-1}}\right| \leq\left|z_{1}\right| \omega_{i}\left(x_{0}, z_{1}, \ldots, \widehat{z}_{i}, \widehat{\Theta}_{0}, \widehat{\Theta}\right) \Theta_{1} .
$$

Based on the completion of squares, it is deduced that there is a smooth function $\omega_{i} \geq 0$ satisfying

$$
\begin{aligned}
& \left|\xi_{i}\right|\left|\frac{\partial z_{i}^{*}}{\partial x_{0}} f_{0}+\frac{\partial \widehat{z}_{i}^{*}}{\partial z_{1}} \frac{f_{1}}{x_{0}^{n-1}}\right| \leq\left[\frac{\sum_{j=1}^{i-1} \xi_{j}^{2}}{2\left(1+\widehat{\Theta}^{2}\right)\left(1+\eta_{i-1}^{2}\right)}+\xi_{i}^{2} \omega\right] \Theta \\
& \leq\left[\frac{\sum_{j=1}^{i-1} \xi_{j}^{2}}{2\left(1+\widehat{\Theta}^{2}\right)\left(1+\eta_{i-1}^{2}\right)}+\xi_{i}^{2} \omega_{i}\right] \widetilde{\Theta} \\
& +\frac{1}{4} \sum_{j=1}^{i-1} \xi_{j}^{2}+\xi_{i}^{2} \omega_{i} \sqrt{\widehat{\Theta}^{2}+1}
\end{aligned}
$$

since

$$
\left|g_{0} \xi_{i-1}\left(\widehat{z}_{i}-\widehat{z}_{i}^{*}\right)\right| \leq \frac{1}{4} \xi_{i-1}^{2}+\xi_{i}^{2} \widetilde{\gamma}_{i}\left(x_{0}, \xi_{1}, \ldots, \xi_{i}, \widehat{\Theta}_{0}, \widehat{\Theta}\right)
$$

for a smooth function $\tilde{\gamma}_{i} \geq 0$.

Substituting (39), (42), and (43) into (36) gives

$$
\begin{aligned}
\dot{V}_{i} \leq & \left(g_{0}+\sum_{k=0}^{i} \varepsilon_{k}^{2}\right)\|\widetilde{z}\|^{2}-\left(n-i+\frac{5}{4}\right)\left(\xi_{1}^{2}+\cdots+\xi_{i-1}^{2}\right) \\
& +\left(\Psi_{i-1}-\dot{\widehat{\Theta}}\right)\left(\widetilde{\Theta}+\eta_{i-1}\right)-g_{0} \xi_{i} \widehat{z}_{i+1} \\
& +\xi_{i}^{2}\left[\omega_{i} \sqrt{\widehat{\Theta}^{2}+1}+\sigma_{i}+\widetilde{\gamma}_{i}\right] \\
& +\left[\frac{\sum_{j=1}^{i-1} \xi_{j}^{2}}{2\left(1+\widehat{\Theta}^{2}\right)\left(1+\eta_{i-1}^{2}\right)}+\xi_{i}^{2} \omega_{i}\right] \widetilde{\Theta}+\xi_{i} \frac{\partial \widehat{z}_{i}^{*}}{\partial \widehat{\Theta}} \dot{\Theta} .
\end{aligned}
$$

Define

$$
\begin{gathered}
\Psi_{i}=\Psi_{i-1}+\frac{\sum_{j=1}^{i-1} \xi_{j}^{2}}{2\left(1+\widehat{\Theta}^{2}\right)\left(1+\eta_{i-1}^{2}\right)}+\xi_{i}^{2} \omega_{i} \\
\eta_{i}=\eta_{i-1}+\xi_{i} \frac{\partial \widehat{z}_{i}^{*}}{\partial \widehat{\Theta}} .
\end{gathered}
$$

Using (34), it is not difficult to verify that

$$
\begin{aligned}
0 & \leq \Psi_{i}\left(x_{0}, \xi_{1}, \ldots, \xi_{i}, \widehat{\Theta}_{0}, \widehat{\Theta}\right) \\
& \leq\left(\xi_{1}^{2}+\cdots+\xi_{i}^{2}\right) \alpha_{i}\left(x_{0}, \xi_{1}, \ldots, \xi_{i}, \widehat{\Theta}_{0}, \widehat{\Theta}\right)
\end{aligned}
$$

for a $C^{\infty} \alpha_{i} \geq 0$. 
Moreover, (44) can be rewritten as follows:

$$
\begin{aligned}
\dot{V}_{i} \leq & \left(g_{0}+\sum_{k=0}^{i} \varepsilon_{k}^{2}\right)\|\widetilde{z}\|^{2}-\left(n-i+\frac{5}{4}\right)\left(\xi_{1}^{2}+\cdots+\xi_{i-1}^{2}\right) \\
& +\left(\Psi_{i}-\dot{\widehat{\Theta}}\right)\left(\widetilde{\Theta}+\eta_{i}\right)-g_{0} \xi_{i} \widehat{z}_{i+1} \\
& +\xi_{i}^{2}\left[\omega_{i} \sqrt{\widehat{\Theta}^{2}+1}+\sigma_{i}+\widetilde{\gamma}_{i}\right] \\
& -\left[\frac{\sum_{j=1}^{i-1} \xi_{j}^{2}}{2\left(1+\widehat{\Theta}^{2}\right)\left(1+\eta_{i-1}^{2}\right)}+\xi_{i}^{2}{\omega_{i}}_{i}\right] \eta_{i-1}-\xi_{i} \frac{\partial \widehat{z}_{i}^{*}}{\partial \widehat{\Theta}} \Psi_{i} .
\end{aligned}
$$

By (46), we have

$$
\begin{aligned}
& \mid\left[\frac{\sum_{j=1}^{i-1} \xi_{j}^{2}}{2\left(1+\widehat{\Theta}^{2}\right)\left(1+\eta_{i-1}^{2}\right)}+\xi_{i}^{2}{\omega_{i}}_{i} \eta_{i-1}+\xi_{i} \frac{\partial \widehat{z}_{i}^{*}}{\partial \widehat{\Theta}} \Psi_{i} \mid\right. \\
& \quad \leq \frac{1}{8} \sum_{j=1}^{i-1} \xi_{j}^{2}+\xi_{i}^{2} \omega_{i} \sqrt{\eta_{i-1}^{2}+1}+\left|\xi_{i} \frac{\partial \widehat{z}_{i}^{*}}{\partial \widehat{\Theta}}\right|\left(\xi_{1}^{2}+\cdots+\xi_{i}^{2}\right) \alpha_{i} \\
& \quad \leq \frac{1}{4} \sum_{j=1}^{i-1} \xi_{j}^{2}+\xi_{i}^{2} \omega_{i} \sqrt{\eta_{i-1}^{2}+1}+\xi_{i}^{2} \sum_{j=1}^{i} \widetilde{\beta}_{i, j} .
\end{aligned}
$$

The last inequality from the following relation

$$
\left|\xi_{i}\right|\left|\xi_{j}^{2} \frac{\partial \widehat{z}_{i}^{*}}{\partial \widehat{\Theta}} \alpha_{i}\right| \leq \frac{1}{8} \xi_{j}^{2}+\xi_{i}^{2} \widetilde{\beta}_{i, j}\left(x_{0}, \xi_{1}, \ldots, \xi_{i}, \widehat{\Theta}_{0}, \widehat{\Theta}\right)
$$

where $\widetilde{\beta}_{i, j} \geq 0,1 \leq j \leq i-1$ are smooth functions and $\widetilde{\beta}_{i, i} \geq\left|\xi_{i}\left(\partial z_{i}^{*} / \partial \widehat{\Theta}\right) \alpha_{i}\right|$, is a smooth function.

Putting (47) and (48) together, we arrive at

$$
\begin{aligned}
\dot{V}_{i} \leq & \left(g_{0}+\sum_{k=0}^{i} \varepsilon_{k}^{2}\right)\|\widetilde{z}\|^{2}-(n-i+1)\left(\xi_{1}^{2}+\cdots+\xi_{i-1}^{2}\right) \\
& +\left(\Psi_{i}-\dot{\widehat{\Theta}}\right)\left(\widetilde{\Theta}+\eta_{i}\right)+g_{0} \xi_{i} \widehat{z}_{i+1} \\
& +\xi_{i}^{2}\left[\omega_{i} \sqrt{\widehat{\Theta}^{2}+1}+\sigma_{i}+\widetilde{\gamma}_{i}+\widehat{\gamma}_{i}\right],
\end{aligned}
$$

where $\widehat{\gamma}_{i}=\Phi_{i} \sqrt{\eta_{i-1}^{2}+1}+\sum_{j=1}^{i} \widetilde{\beta}_{i, j}\left(x_{0}, \xi_{1}, \ldots, \xi_{i}, \widehat{\Theta}_{0}, \widehat{\Theta}\right)$.

Now, it easy to see that the smooth virtual controller

$$
\begin{gathered}
\widehat{z}_{i+1}^{*}=-\frac{1}{g_{0}} \xi_{i}\left[n-i+1+\kappa_{i}\left(x_{0}, \xi_{1}, \ldots, \xi_{i}, \widehat{\Theta}_{0}, \widehat{\Theta}\right)\right] \\
:=\xi_{i} \beta_{i}\left(x_{0}, \xi_{1}, \ldots, \xi_{i}, \widehat{\Theta}_{0}, \widehat{\Theta}\right) \\
\kappa_{i}=\omega_{i} \sqrt{\widehat{\Theta}^{2}+1}+\sigma_{i}+\widetilde{\gamma}_{i}+\widehat{\gamma}_{i}
\end{gathered}
$$

renders

$$
\begin{aligned}
\dot{V}_{i} \leq & \left(g_{0}+\sum_{k=0}^{i} \varepsilon_{k}^{2}\right)\|\widetilde{z}\|^{2}-(n-i+1)\left(\xi_{1}^{2}+\cdots+\xi_{i}^{2}\right) \\
& +g_{0} \xi_{i}\left(\widehat{z}_{i+1}-\widehat{z}_{i+1}^{*}\right)+\left(\Psi_{i}-\dot{\widehat{\Theta}}\right)\left(\widetilde{\Theta}+\eta_{i}\right) .
\end{aligned}
$$

As $i=n$, the last step, we can construct explicitly a change of coordinates $\left(\xi_{1}, \ldots, \xi_{n}\right)$, a positive-definite and proper Lyapunov function $V_{n}=V_{n-1}+\xi_{n}^{2} / 2$, and a smooth controller $u_{1}^{*}=-\xi_{n} \beta_{n}$ such that

$$
\begin{aligned}
\dot{V}_{n} \leq & \left(g_{0}+\sum_{k=0}^{n} \varepsilon_{k}^{2}\right)\|\widetilde{z}\|^{2}-\left(\xi_{1}^{2}+\cdots+\xi_{n}^{2}\right) \\
& +\xi_{n}\left(u_{1}-u_{1}^{*}\right)+\left(\Psi_{n}-\dot{\hat{\Theta}}\right)\left(\widetilde{\Theta}+\eta_{n}\right) .
\end{aligned}
$$

Therefore, by choosing the smooth actual control $u_{1}$ and update law for $\widehat{\Theta}$ as

$$
\begin{gathered}
u_{1}=-\xi_{n} \beta_{n}\left(x_{0}, \xi_{1}, \ldots, \xi_{i}, \widehat{\Theta}_{0}, \widehat{\Theta}\right) \\
\dot{\widehat{\Theta}}=\Psi_{n}\left(x_{0}, \xi_{1}, \ldots, \xi_{i}, \widehat{\Theta}_{0}, \widehat{\Theta}\right)
\end{gathered}
$$

we have

$$
\dot{V}_{n} \leq\left(g_{0}+\sum_{k=0}^{n} \varepsilon_{k}^{2}\right)\|\widetilde{z}\|^{2}-\left(\xi_{1}^{2}+\cdots+\xi_{n}^{2}\right) .
$$

In Section 4, we will show that an appropriate choice of the design parameters $k_{0}$ and $\varepsilon_{i}$ guarantees the asymptotic convergence of the state $\left(x_{0}, x\right)$ of the original system (1) in closed loop with our control laws (7), (8), (54), and (55) as long as the initial condition $x_{0}\left(t_{0}\right)$ is nonzero. It will also be shown in the next section how to handle the singular case where $x_{0}\left(t_{0}\right)$ is zero.

\section{Switching Controller and Main Result}

In the preceding section, we have given controller design for $x_{0}\left(t_{0}\right) \neq 0$. Without loss of generality, we can assume that $t_{0}=0$. Now, we discuss how to select the control laws $u_{0}$ and $u_{1}$ when $x_{0}(0)=0$. Choose $u_{0}$ as

$$
u_{0}=x_{0} g_{0}+u_{0}^{*}, \quad 2 u_{0}^{*}>0,
$$

where $g_{0}$ is given by (7).

Choosing the same Lyapunov function (9), its time derivative is given by

$$
\dot{V}_{0} \leq-k_{0} x_{0}^{2}+u_{0}^{*} x_{0}
$$

which leads to the boundedness of $x_{0}$ and consequently the bound of $\widehat{\Theta}_{0}$ as well. Consequently, $x_{0}$ does not escape and $x_{0}\left(t_{s}\right) \neq 0$ for any given finite $t_{s}>0$. Thus state-scaling for the control design can be carried out.

During the time period $\left[0, t_{s}\right)$, using $u_{0}$ defined in (57), new control law $u_{1}=u_{1}^{*}\left(x_{0}, x, \mu\right)$ and new update law 
$\dot{\mu}=\nu^{*}\left(x_{0}, x, \mu\right)$ can be obtained by following the control procedure described above to the original $x$-system in (1). Then we can conclude that the $x$-state of (1) cannot blow up during the time period $\left[0, t_{s}\right)$. Since $x\left(t_{s}\right) \neq 0$ at $t_{s}$, we can switch the control input $u_{0}$ and $u_{1}$ to (7) and (54), respectively.

We are now ready to state the main theorem of our paper.

Theorem 8. Under Assumption 1, if the above switching control strategy is applied to (1) with an appropriate choice of the design parameters $k_{i}$ and $\varepsilon_{i}^{\prime} s$, uncertain system (1) is globally regulated at the origin. Furthermore, the estimated parameters are bounded.

Proof. According to the above analysis, it suffices to prove the statement in the case where $x_{0}(0) \neq 0$.

Since we have already proven that $\lim _{t \rightarrow \infty} x_{0}(t)=0$ and $\widehat{\Theta}_{0}$ is bounded in Section 3, we just need to show that $\lim _{t \rightarrow \infty} x(t)=0$ and $\widehat{\Theta}$ is bounded. In this case, as a result of (7), (11), (54), and (55), from (56), it holds that

$$
\dot{V}_{n} \leq\left(g_{0}+\sum_{k=0}^{n} \varepsilon_{k}^{2}\right)\|\tilde{z}\|^{2}-\left(\xi_{1}^{2}+\cdots+\xi_{n}^{2}\right) .
$$

Choose the design parameters $k_{0}$ and $\varepsilon_{i}^{\prime} s$ so that

$$
k_{0} \geq \sum_{k=0}^{n} \varepsilon_{k}^{2}
$$

Therefore, we have

$$
\dot{V}_{n} \leq-\left(\xi_{1}^{2}+\cdots+\xi_{n}^{2}\right)
$$

which means $\xi$ and $\widehat{\Theta}$ are bounded. Furthermore, we can conclude that $\xi \rightarrow 0$ as $t \rightarrow \infty$ by using LaSalle's Invariant Theorem [26]. This implies that $z \rightarrow 0$ as $t \rightarrow \infty$. From the state scaling transformation (15), we conclude that $x \rightarrow 0$ as $t \rightarrow \infty$. This completes the proof of Theorem 8 .

Remark 9. As seen from (54) and (7), the control law $u_{1}$ may exhibit extremely large value when $x_{0}\left(t_{0}\right) \neq 0$ is sufficiently small. This is unacceptable from a practical point of view. It is therefore recommended to apply (57) in order to enlarge the initial value of $x_{0}$ before we appeal to the asymptotically converging controllers (7) and (54).

\section{Example}

To verify our proposed controller, we consider the following low-dimensional system with parametric uncertainty:

$$
\begin{gathered}
\dot{x}_{0}=u_{0} \\
\dot{x}_{1}=u_{0} x_{2}+\ln \left(1+\left(\theta x_{1}\right)^{2}\right) \\
\dot{x}_{2}=u_{1},
\end{gathered}
$$

where $\theta$ is unknown constant parameter. Define $\Theta=1+$ $\theta^{2}$; obviously, this system satisfies Lemma 4 ; that is, $\mid \ln (1+$ $\left.\left(\theta x_{1}\right)^{2}\right)|\leq| x_{1} \mid \Theta$. The purpose is to design $u_{0}$ and $u_{1}$ based on only $y=\left(x_{0}, x_{1}\right)^{T}$ such that $\left(x_{0}, x_{1}, x_{2}\right)^{T} \rightarrow 0$ as $t \rightarrow \infty$.
If $x(0)=0$, controls $u_{0}$ and $u_{1}$ are set as in Section 4 in interval $\left[0, t_{s}\right)$, such that $x\left(t_{s}\right) \neq 0$; then we can adopt the controls developed below. Therefore, without loss of generality, we assume that $x(0) \neq 0$.

For simplicity, in the first subsystem, we can choose the control law $u_{0}=g_{0} x_{0}$ and introduce the introduce the state scaling transformation:

$$
z_{1}=\frac{x_{1}}{x_{0}}, \quad z_{2}=x_{2}
$$

In new $z$-coordinates, the $\left(x_{1}, x_{2}\right)$-subsystem of $(62)$ is rewritten as

$$
\begin{gathered}
\dot{z}_{1}=g_{0}\left(z_{2}-z_{1}\right)+\frac{1}{x_{0}} \ln \left(1+\left(\theta x_{1}\right)^{2}\right) \\
\dot{z}_{2}=u_{1} .
\end{gathered}
$$

Design observer to reconstruct $z_{1}$ and $z_{2}$ of system (62) as follows:

$$
\begin{gathered}
\dot{\vec{z}}_{1}=g_{0}\left(\widehat{z}_{2}-\widehat{z}_{1}\right)+g_{0} k_{1}\left(z_{1}-\widehat{z}_{1}\right), \quad k_{1}=-4 \\
\dot{\vec{z}}_{2}=u_{1}+k_{2}\left(z_{1}-\widehat{z}_{1}\right), \quad k_{2}=-2 .
\end{gathered}
$$

Then, the estimation error $\widetilde{z}=\left(z_{1}-\widehat{z}_{1}, z_{2}-\widehat{z}_{2}\right)^{T}$ satisfies the equation

$$
\dot{\tilde{z}}=g_{0}\left(\begin{array}{cc}
-k_{1}-1 & 1 \\
k_{2} & 0
\end{array}\right) \tilde{z}+\frac{1}{x_{0}}\left(\begin{array}{c}
\ln \left(1+\left(\theta x_{1}\right)^{2}\right) \\
0
\end{array}\right) .
$$

In this case, we have

$$
A=\left(\begin{array}{cc}
3 & 1 \\
-2 & 0
\end{array}\right)
$$

Solving the matrix equation $A^{T} P+P A=I_{2}$, we obtain

$$
P=\left(\begin{array}{ll}
0.500 & 0.500 \\
0.500 & 0.100
\end{array}\right) \text {. }
$$

The eigenvalues of $P$ are $0.1910,1.3090$, and thus $\|P\|=$ 1.3090

Using the method given in Section 3, we can design the adaptive controller as follows:

$$
\begin{gathered}
\widehat{z}_{2}^{*}=-\frac{1}{g_{0}} z_{1}\left[3+g_{0}^{2} \varepsilon_{1}^{-2}+1.604 \varepsilon_{0}^{-2}+\sqrt{\widehat{\Theta}^{2}+1}\right]=z_{1} \beta_{1} \\
u_{1}=-\xi_{2}\left[9+\beta_{1}+\beta_{1}^{4}+\beta_{1}^{2} \sqrt{\widehat{\Theta}^{2}+1}\right] \\
\dot{\widehat{\Theta}}=z_{1}^{2}\left(1.604 \varepsilon_{0}^{-2}+1\right)^{2}+\xi_{2}^{2} \beta_{1}^{2} .
\end{gathered}
$$

The unknown parameter $\theta$ is assumed to be 1 . In simulation, the design parameters are chosen as $g_{0}=-1$ and $\varepsilon_{0}=$ $\varepsilon_{1}=\varepsilon_{2}=0.25$. The simulation results for initial conditions $\left(x_{0}(0), x_{1}(0), x_{2}(0)\right)=(2,1,-1)$ and $\left(\widehat{z}_{1}(0), \widehat{z}_{2}(0), \widehat{\Theta}(0)\right)=$ $(1,-1,1)$ are shown in Figures $1-4$. From the figures, it is clear to see all the closed-loop system states are regulated to zero and the parameter estimates are bounded as proven in Theorem 8. 


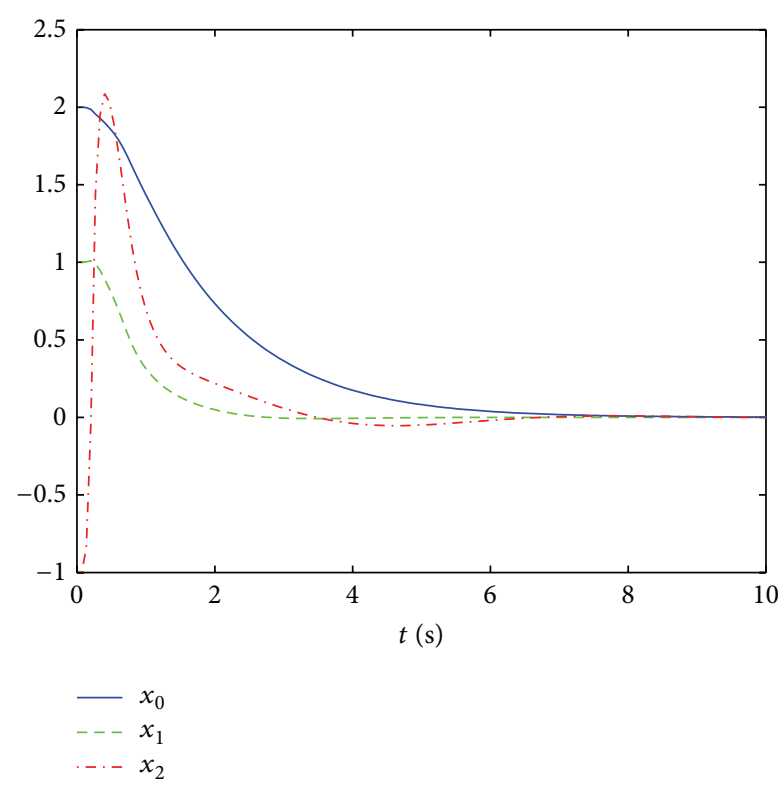

FIGURE 1: System states.

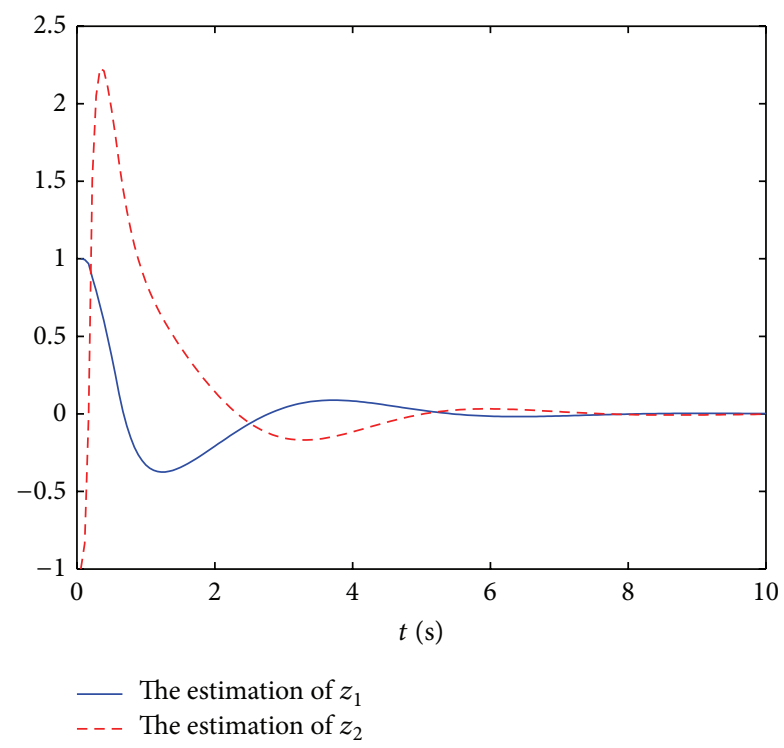

Figure 2: Observer states.

\section{Conclusions}

In this paper, a constructive adaptive output feedback control strategy is presented for a class of nonlinearly parameterized nonholonomic systems with strong nonlinear drifts. To deal with the nonlinear parameterization problem, a parameter separation technique is introduced to transform the nonlinear parameterized nonholonomic system into a linearlike parameterized nonholonomic system. We estimate only $\Theta$, the bound of the unknown parameter rather than the parameter vector $\theta$. This, in turn, results in a minimum dimension (1D) parameter update law. The controller design is developed by using the integrator backstepping approach

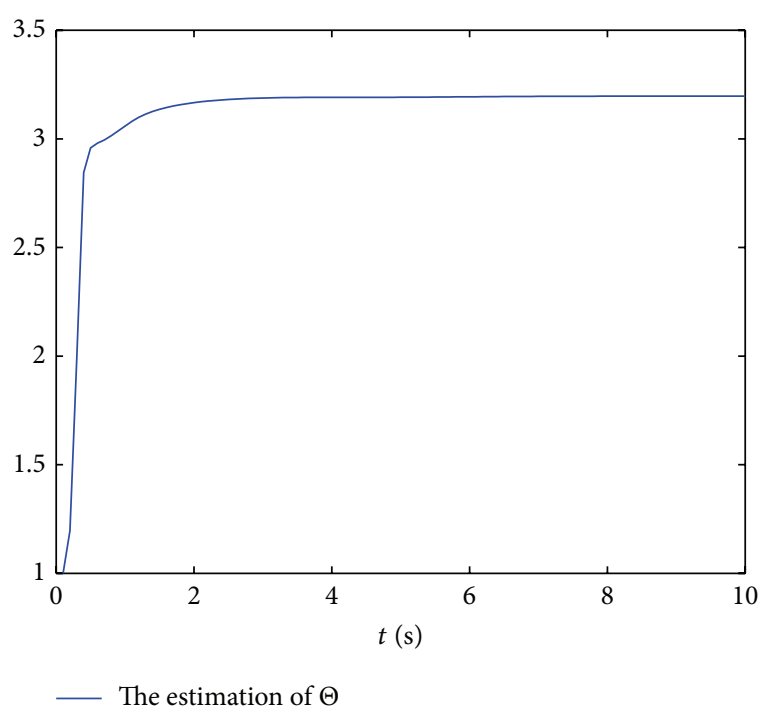

Figure 3: Parameter estimate.

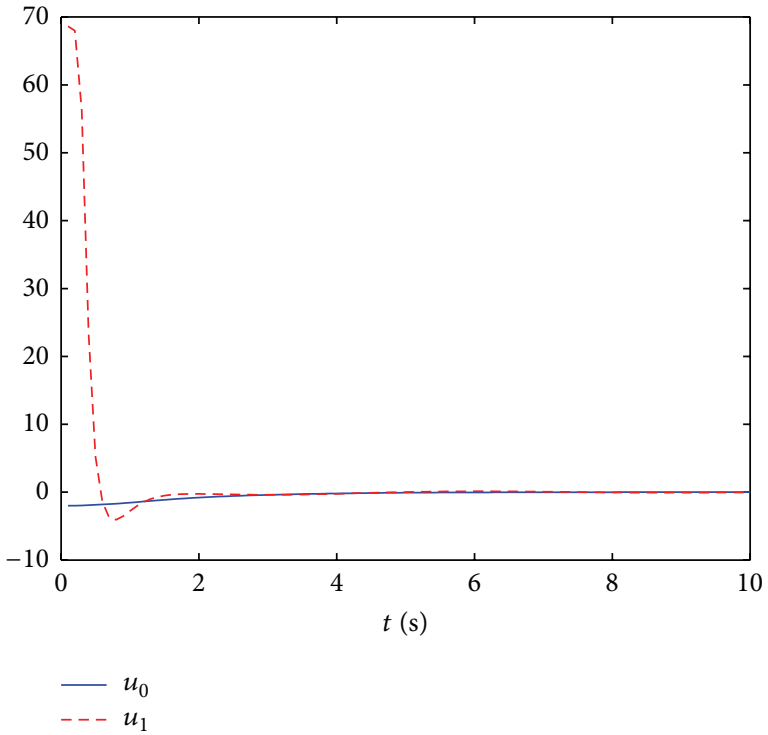

Figure 4: Control inputs.

based on observer and parameter estimator. With a switching strategy eliminating the phenomenon of uncontrollability, the system states asymptotically converge to the origin, while other signals remaining bounded is guaranteed. Simulation results have shown the effectiveness and feasibility of the proposed control strategy.

\section{Conflict of Interests}

The authors declare that there is no conflict of interests regarding the publication of this paper. 


\section{Acknowledgments}

The authors thank the editor and the anonymous reviewers for their constructive comments and suggestions for improving the quality of the paper. This work has been supported in part by National Nature Science Foundation of China under Grant no. 61073065 and the Key Program of Science Technology Research of Education Department of Henan Province under Grants nos. 13A120016, 14A520003.

\section{References}

[1] R. M. Murray and S. S. Sastry, "Nonholonomic motion planning: steering using sinusoids," IEEE Transactions on Automatic Control, vol. 38, no. 5, pp. 700-716, 1993.

[2] R. W. Brockett, "Asymptotic stability and feedback stabilization," in Differ. Geom. Control Theory, R. W. Brockett, R. S. Millman, and H. J. Sussmann, Eds., pp. 2961-2963, 1983.

[3] I. Kolmanovsky and N. H. McClamroch, "Developments in nonholonomic control problems," IEEE Control Systems Magazine, vol. 15, no. 6, pp. 20-36, 1995.

[4] A. Astolfi, "Discontinuous control of nonholonomic systems," Systems \& Control Letters, vol. 27, no. 1, pp. 37-45, 1996.

[5] Z. Jiang, "Iterative design of time-varying stabilizers for multiinput systems in chained form," Systems \& Control Letters, vol. 28, no. 5, pp. 255-262, 1996.

[6] W. L. Xu and W. Huo, "Variable structure exponential stabilization of chained systems based on the extended nonholonomic integrator," Systems \& Control Letters, vol. 41, no. 4, pp. 225-235, 2000 .

[7] Z. Sun, S. S. Ge, W. Huo, and T. H. Lee, "Stabilization of nonholonomic chained systems via nonregular feedback linearization," Systems \& Control Letters, vol. 44, no. 4, pp. 279289, 2001

[8] Y. Tian and S. Li, "Exponential stabilization of nonholonomic dynamic systems by smooth time-varying control," Automatica, vol. 38, no. 7, pp. 1139-1146, 2002.

[9] Z. Jiang, "Robust exponential regulation of nonholonomic systems with uncertainties," Automatica, vol. 36, no. 2, pp. 189209, 2000.

[10] Z. Xi, G. Feng, Z. P. Jiang, and D. Cheng, "A switching algorithm for global exponential stabilization of uncertain chained systems," IEEE Transactions on Automatic Control, vol. 48, no. 10, pp. 1793-1798, 2003.

[11] Z. R. Xi, G. Feng, Z. P. Jiang, and D. Z. Cheng, "Output feedback exponential stabilization of uncertain chained systems," Journal of the Franklin Institute, vol. 344, no. 1, pp. 36-57, 2007.

[12] K. D. Do and J. Pan, "Adaptive global stabilization of nonholonomic systems with strong nonlinear drifts," Systems \& Control Letters, vol. 46, no. 3, pp. 195-205, 2002.

[13] S. S. Ge, Z. P. Wang, and T. H. Lee, "Adaptive stabilization of uncertain nonholonomic systems by state and output feedback," Automatica. A Journal of IFAC, The International Federation of Automatic Control, vol. 39, no. 8, pp. 1451-1460, 2003.

[14] Y. Hu, S. S. Ge, and C. Su, "Stabilization of uncertain nonholonomic systems via time-varying sliding mode control," IEEE Transactions on Automatic Control, vol. 49, no. 5, pp. 757-763, 2004.

[15] Y. Q. Wu, Y. Zhao, and J. B. Yu, "Global asymptotic stability controller of uncertain nonholonomic systems," Journal of the
Franklin Institute. Engineering and Applied Mathematics, vol. 350, no. 5, pp. 1248-1263, 2013.

[16] Y. G. Liu and J. F. Zhang, "Output-feedback adaptive stabilization control design for non-holonomic systems with strong non-linear drifts," International Journal of Control, vol. 78, no. 7, pp. 474-490, 2005.

[17] X. Y. Zheng and Y. Q. Wu, "Adaptive output feedback stabilization for nonholonomic systems with strong nonlinear drifts," Nonlinear Analysis. Theory, Methods \& Applications. An International Multidisciplinary Journal. Series A: Theory and Methods, vol. 70, no. 2, pp. 904-920, 2009.

[18] Y. Wu, G. Ju, and X. Zheng, "Adaptive output feedback control for nonholonomic systems with uncertain chained form," International Journal of Systems Science, vol. 41, no. 12, pp. 1537-1547, 2010.

[19] G. L. Ju, Y. Q. Wu, and W. H. Sun, "Output-feedback control for nonholonomic systems with linear growth condition," Journal of Systems Science \& Complexity, vol. 24, no. 5, pp. 862-874, 2011.

[20] Y. Zhao and Y. Q. Wu, "Control of nonholonomic systems with nonlinear unmeasured dynamics by output feedback," Journal of Control Theory and Applications, vol. 11, no. 3, pp. 504-512, 2013.

[21] F. Z. Gao, F. S. Yuan, and H. J. Yao, "Robust adaptive control for nonholonomic systems with nonlinear parameterization," Nonlinear Analysis: Real World Applications, vol. 11, no. 4, pp. 3242-3250, 2010.

[22] F. Z. Gao, F. S. Yuan, H. J. Yao, and X. W. Mu, "Adaptive stabilization of high order nonholonomic systems with strong nonlinear drifts," Applied Mathematical Modelling. Simulation and Computation for Engineering and Environmental Systems, vol. 35, no. 9, pp. 4222-4233, 2011.

[23] F. Z. Gao, Y. L. Shang, and F. S. Yuan, "Robust adaptive finitetime stabilization of nonlinearly parameterized nonholonomic systems," Acta Applicandae Mathematicae, vol. 123, no. 1, pp. 157-173, 2013.

[24] W. Lin and C. Qian, "Adaptive control of nonlinearly parameterized systems: a nonsmooth feedback framework," IEEE Transactions on Automatic Control, vol. 47, no. 5, pp. 757-774, 2002.

[25] E. G. Sontag, "Smooth stabilization implies coprime factorization," IEEE Transactions on Automatic Control, vol. 34, no. 4, pp. 435-443, 1989.

[26] H. K. Khalil, Nonlinear Systems, Prentice-Hall, Upper Saddle River, NJ, USA, 3rd edition, 2002. 


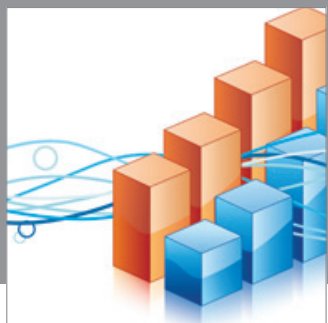

Advances in

Operations Research

mansans

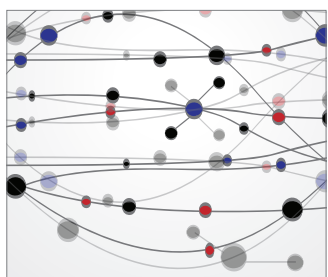

The Scientific World Journal
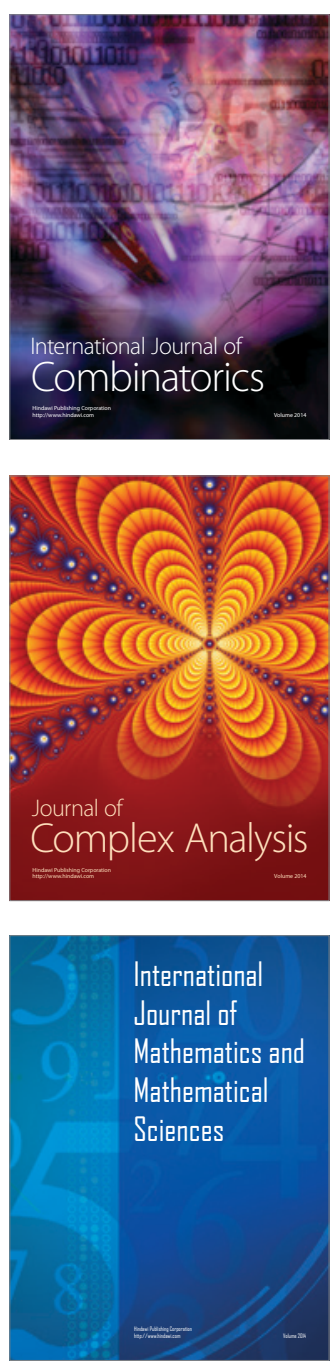
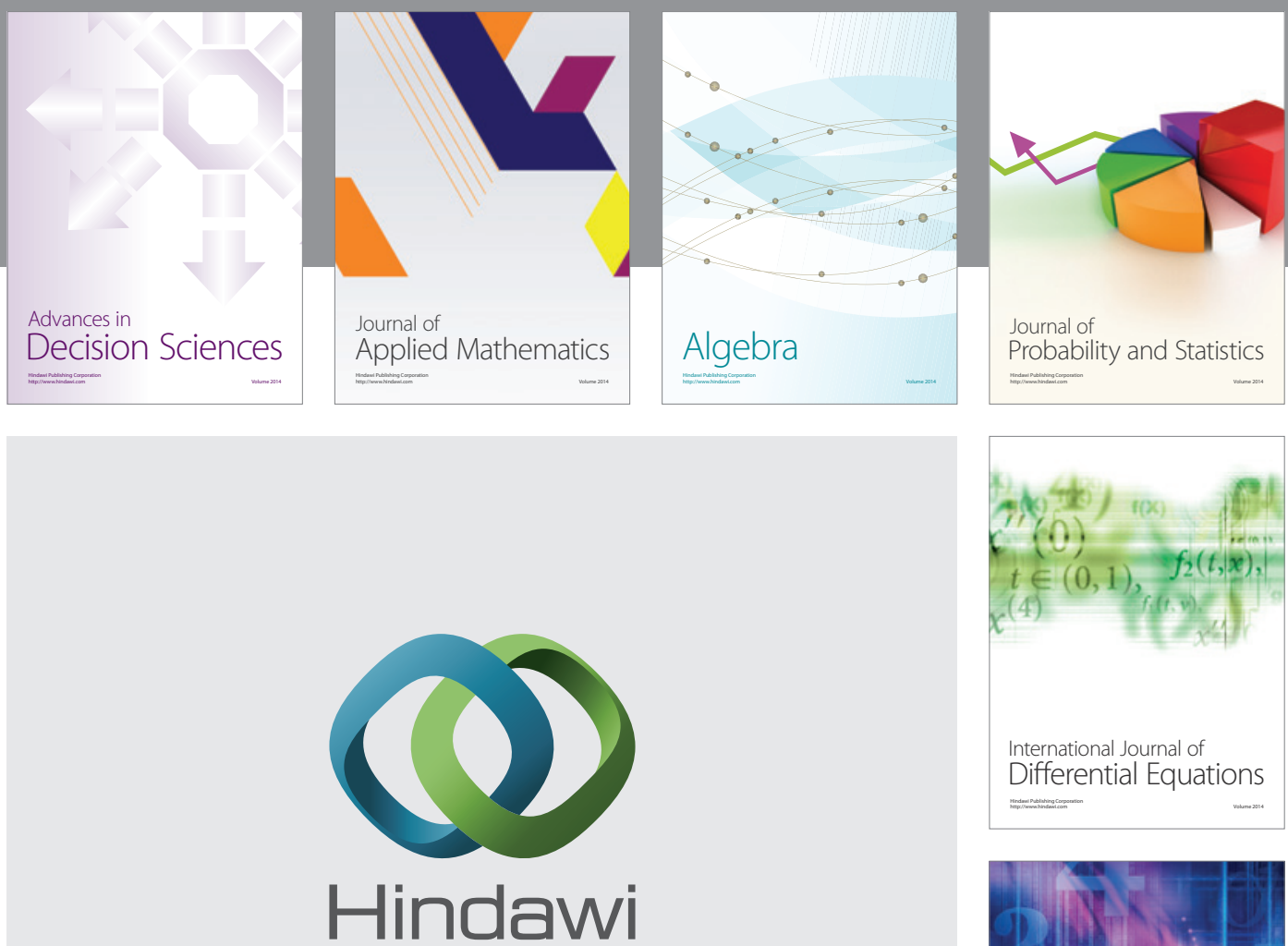

Submit your manuscripts at http://www.hindawi.com
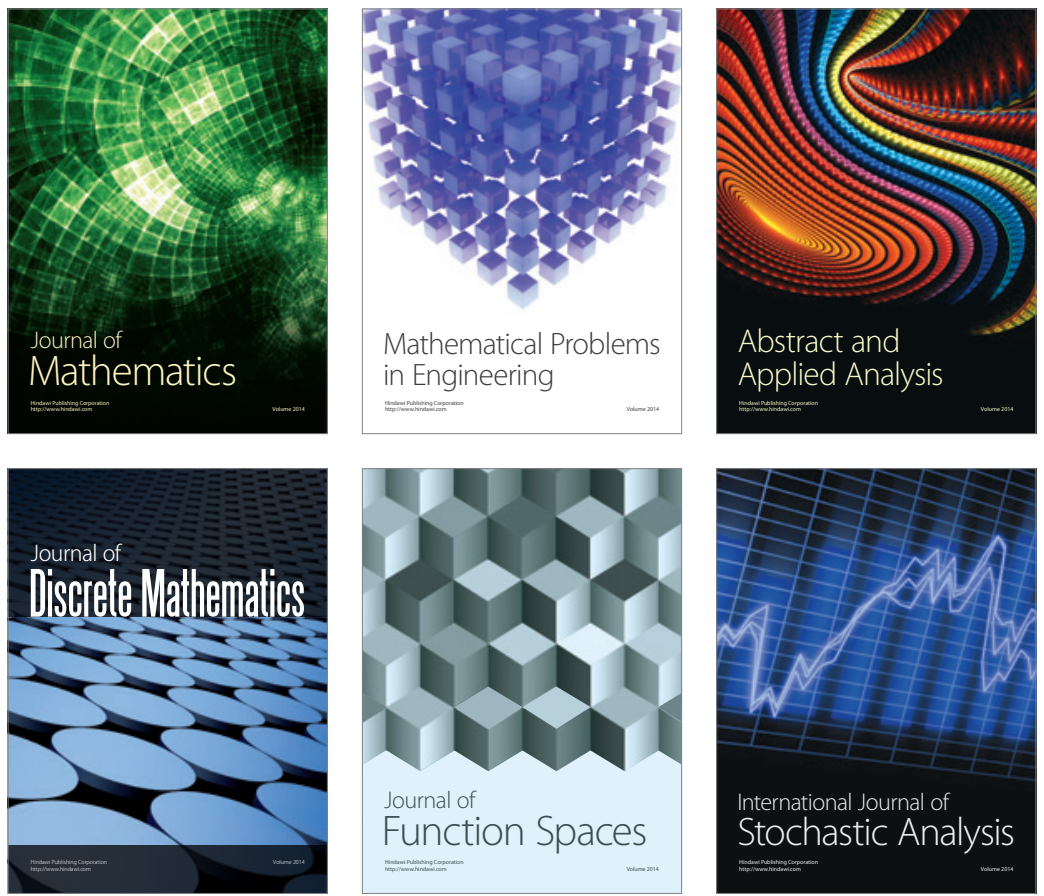

Journal of

Function Spaces

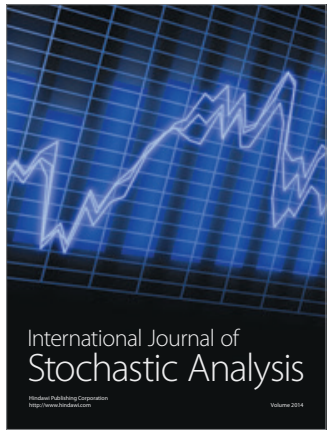

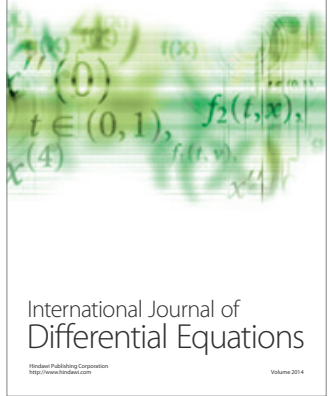
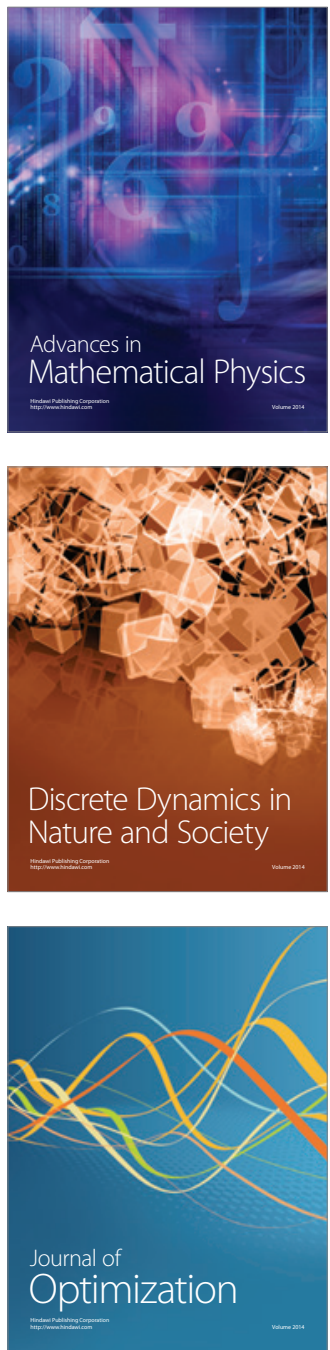